\title{
Optically pumped high-power semiconductor disk laser with gain element engineered for wide tunability
}

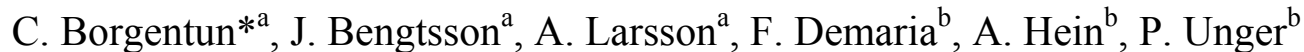 \\ ${ }^{a}$ Photonics Laboratory, Department of Microtechnology and Nanoscience (MC2), \\ Chalmers University of Technology, SE-412 96 Göteborg, Sweden \\ ${ }^{\mathrm{b}}$ Institute of Optoelectronics, Ulm University, 89081 Ulm, Germany
}

\begin{abstract}
The layer structure of the gain element in an optically pumped semiconductor disk laser (OP-SDL) was designed for wide tunability. This was achieved by a parametric optimization of the structure, which in effect balanced the spectrally varying influence of the gain of the quantum wells, the longitudinal distribution of the standing wave lasing field in the structure, and the degree of resonance in the subcavity formed between the distributed Bragg reflector at the bottom and the air-semiconductor interface at the top. The quality measure in the optimization was the spectral reflectance of the gain element for light incident from the external cavity at low power. This unsaturated reflectance was compared to its target function, which was constant at a specified value larger than unity over a wide, prescribed wavelength range. The fabricated gain element was used in a linear OP-SDL with a rotatable intra-cavity birefringent filter for wavelength tuning. The design principles for achieving wide tunability were experimentally validated by the strong agreement between measurements and simulations of the spectral threshold pump intensity. Furthermore, tuning experiments at high pump powers were performed showing that the lasing wavelength could be tuned from $967 \mathrm{~nm}$ to $1010 \mathrm{~nm}$ with a maximum output power of $2.6 \mathrm{~W}$.
\end{abstract}

Keywords: optically pumped semiconductor disk laser, OP-SDL, vertical external cavity surface-emitting laser, VECSEL, design, tuning, wide tunability, high power.

\section{INTRODUCTION}

A majority of the semiconductor lasers found in technologically important applications are either edge-emitting lasers or vertical cavity surface-emitting lasers (VCSELs). The edge-emitting lasers can emit beams of high output power but the shapes of the beams are highly elliptical and require sophisticated external optics for efficient use in many applications. A VCSEL beam on the other hand can be close to circular and diffraction-limited but cannot deliver high output power. An optically pumped semiconductor disk laser (OP-SDL), or vertical external cavity surface-emitting laser (VECSEL) as it is also referred to, can combine the high output power of the edge-emitting devices with the superior beam quality of the VCSELs ${ }^{1-3}$. Another advantage of the OP-SDL is its free-space cavity, which allows for the convenient insertion of various optical elements, such as non-linear crystals for frequency doubling, semiconductor saturable absorber mirrors (SESAMs) for mode-locking or wavelength-selective elements for wavelength tuning.

Although applications such as intra-cavity laser absorption spectroscopy (ICLAS) might greatly benefit from a widely tunable OP-SDL with sufficient output power, research in extending the tuning range has only recently been given major attention. Pioneering efforts were made by Garnache et al. applying a principle for designing the gain element, in which the wavelength dependent effects of subcavity resonance and material gain were balanced ${ }^{4}$. There has also been work employing multi-chip setups with record-high output power but with limited tuning range ${ }^{5}$. Recent work used a set of heterogeneous quantum wells (QWs) resulting in a very wide tuning range but low output power ${ }^{6}$.

This work has investigated whether the tuning range can be extended while maintaining high output power, which further should be only slowly varying with the lasing wavelength. The design principle of this work builds on the one used by Garnache et al., but the design of the gain element was extended to include a much longer subcavity with 12 identical QWs. In this way most of the pump light will be absorbed and more optical gain will be provided. Also, part of the structure was parametrically optimized to even more accurately control the broadband properties.

*carl.borgentun@chalmers.se; phone +46-31-7721607; fax +46-31-7721540; chalmers.se/mc2

Semiconductor Lasers and Laser Dynamics IV, edited by Krassimir Panayotov,

Marc Sciamanna, Angel A. Valle, Rainer Michalzik, Proc. of SPIE Vol. 7720, 772014

(C) 2010 SPIE · CCC code: 0277-786X/10/\$18 · doi: 10.1117/12.854129

Proc. of SPIE Vol. $7720772014-1$ 


\section{DESIGN OF THE GAIN ELEMENT}

The gain element consists of a subcavity formed by the layers between the air-semiconductor interface and the distributed Bragg reflector (DBR). The subcavity contains not only the active region, i.e. the gain structure with the QWs, but also an anti-reflection (AR) structure next to the air interface.

The gain element was optimized by considering the unsaturated reflectance for an incident weak external cavity field in a certain wavelength range. As a quality measure of a certain gain element design, its reflectance spectrum under pumping was calculated and compared to a target reflectance spectrum, which was specified as a top-hat function with an upper value of 1.03 to approximately compensate for the assumed optical losses of the cavity. The lower reflectance level of the target spectrum was specified to be 1.00, which is the approximate reflectance of the stopband of the DBR mirror. The width of the top-hat is roughly equivalent to the tuning range and was to be maximized under the given conditions.

The unsaturated reflectance of the gain element was calculated by the use of a conventional transfer matrix model (TMM) for the field propagation to simulate the optical field inside the subcavity. The QW gain was included in the imaginary part of the refractive index and was calculated from a physical model including effects from QW strain, lifting of valence band degeneracy, and Coulomb interaction. The gain model further assumed exponential pump light absorption in the barriers and used the phenomenological $\mathrm{ABC}$ recombination law to calculate the population densities of the QWs.

One of the boundary conditions of the optimization problem was that the QWs should be homogeneously populated such that the carrier density in each QW would be high enough for optical amplification. For this reason, diffusion barriers with higher bandgap energy ${ }^{7}$ were included to create well-defined absorption volumes for the separate groups of QWs. Within such a volume the generated charge carriers were assumed to be trapped with equal probability by any of the QWs. Another boundary condition was that the number of QWs should be 12, which is a trade-off between optical gain and efficient heat extraction. Including more QWs would expand the length of the gain medium and provide more gain. On the other hand, more QWs require more absorption volumes that would have to be quite long since the remaining pump intensity would be quite low, which would inhibit the heat transfer to the heatsink. A final but essential requirement was that the QWs should be aligned with antinodes of the standing wave of the subcavity optical field at the antiresonant (center) wavelength at $980 \mathrm{~nm}$.

The optimization parameters were the distribution of QWs and the parameters of the AR structure, i.e. the number of layers in the AR structure and the compositions and thicknesses of these layers. Setting the number of QWs positioned at each antinode also determines the length of the active region since the requirement for QW population homogeneity in turn determines the sizes of the absorption volumes between the diffusion barriers. Having done this, the AR structure can be optimized.

The main purpose of the AR structure is to balance the large increase in subcavity field intensity near resonance. Roughly, the design of the AR structure is similar to a DBR with pairs of $\sim \lambda / 4$ thick layers with alternating high and low refractive index, but the AR structure ends with a $\sim \lambda / 2$ thick layer, which causes the AR effect by destructive interference. The number of layer pairs, the chemical composition of the high index layers, and the value for the center wavelength were optimized to fine-tune the reflectance spectrum. The first two parameters control the strength of the AR effect and the last determines the spectral position. For the reflectance spectrum, this implies that a higher, flatter, and more symmetrical spectral reflectivity curve can be achieved. The effects of varying the parameters of this AR structure can be directly seen in figures 1-3. If the AR structure is not included the reflectance spectrum shows two significant peaks near the resonance wavelengths at 960 and $1000 \mathrm{~nm}$, and it is lower than the desired target value of 1.03 for all wavelengths within the specified bandwidth, see figure 1, curve 1E. 


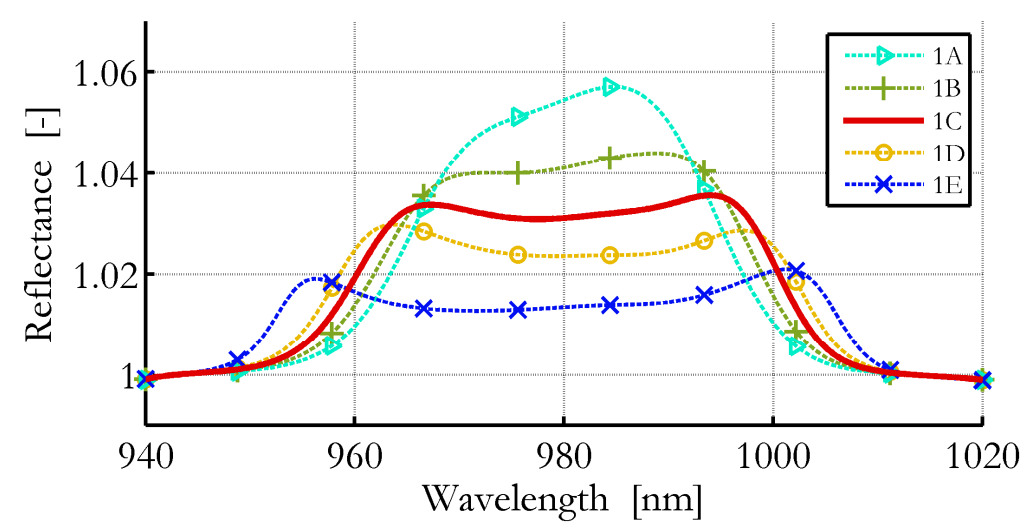

Figure 1. Simulated reflectance spectra from the AR structure optimization process when varying the number of layer pairs: 1A) 4.5 pairs, 1B) 3.5 pairs, 1C) 2.5 pairs, 1D) 1.5 pairs, and 1E) without AR structure. The high index layers contain $20 \%$ aluminum and the center wavelength is $968 \mathrm{~nm}$ for all cases. Curve $1 \mathrm{C}$ is the spectrum for the device designed in this work.

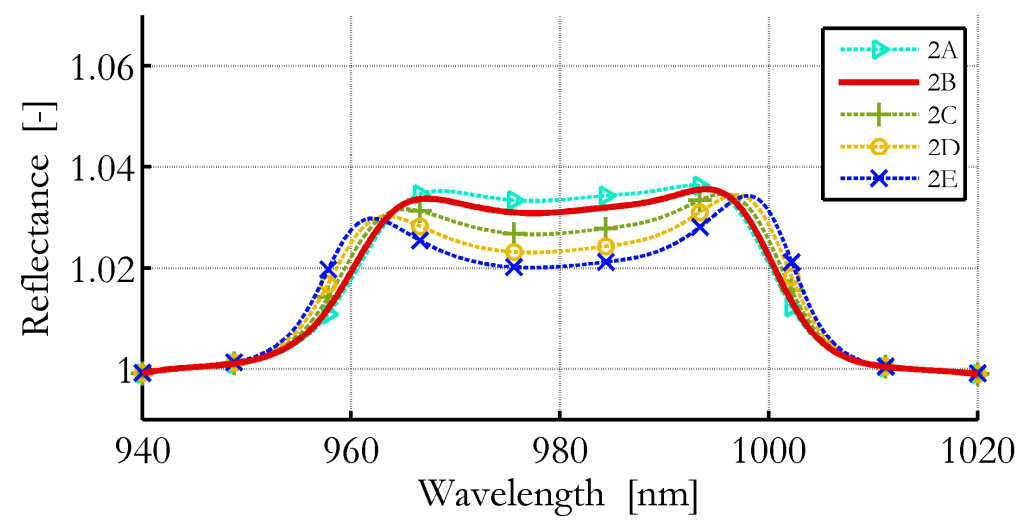

Figure 2. Simulated reflectance spectra from the AR structure optimization process when varying the composition of the high index layers: 2A) $10 \%$ aluminum, 2B) $20 \%$ aluminum, 2C) $40 \%$ aluminum, 2D) $60 \%$ aluminum, and 2E) $80 \%$ aluminum. The number of layer pairs is 2.5 and the center wavelength is $968 \mathrm{~nm}$ for all cases. Curve $2 \mathrm{~B}$ is the spectrum for the device designed in this work.

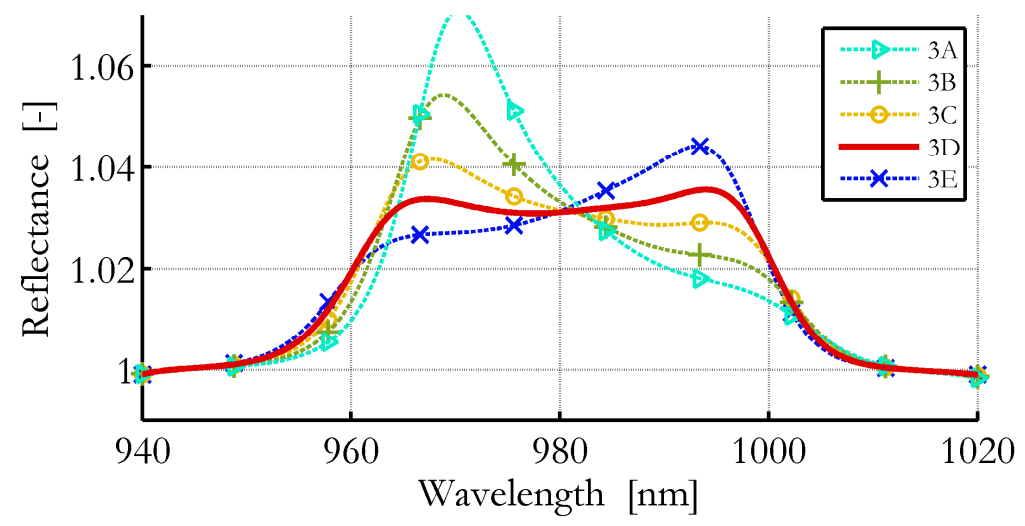

Figure 3. Simulated reflectance spectra from the AR structure optimization process when varying the center wavelength of the AR structure: 3A) $995 \mathrm{~nm}, 3 \mathrm{~B}) 985 \mathrm{~nm}, 3 \mathrm{C}) 975 \mathrm{~nm}, 3 \mathrm{D}) 968 \mathrm{~nm}, 3 \mathrm{E}) 960 \mathrm{~nm}$. The high index layers contain 20\% aluminum and the number of layer pairs is 2.5 for all cases. Curve $3 \mathrm{D}$ is the spectrum for the device designed in this work. 
The gain element was optimized for groups of one, two, and three QWs at each antinode, see figure 4. From this it was decided to use the design with QWs grouped in pairs, i.e. two at each antinode, for the fabrication and experimental characterization. The AR structure of this design, with its parameters optimized as described above, had 2.5 pairs of $\mathrm{Al}(20 \%) \mathrm{Ga}(80 \%)$ As layers with thicknesses according to a center wavelength of $968 \mathrm{~nm}$. The structure also included GaAsP layers with tensile strain to compensate for the compressive strain from the InGaAs QWs. The main features of the optimized structure and the optical field intensities at the antiresonant and at one of the resonant wavelengths can be seen in figure 5. The simulated reflectance spectrum of the optimized design had a reflectance above $\sim 1.03$ over a $34 \mathrm{~nm}$ wavelength range.

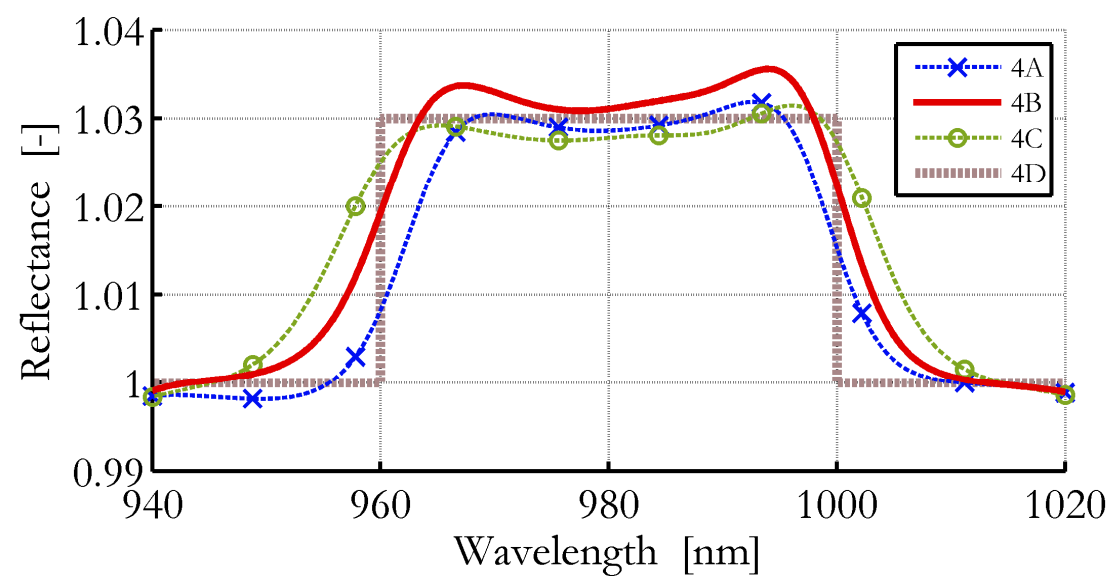

Figure 4. Simulated reflectance spectra for gain elements designed with different sizes of the groups of QWs at each subcavity field antinode. Curves 4A, 4B, and 4C are simulated for groups of one, two, and three QWs, respectively. The pump intensity is the same for all cases and chosen such that the assumed approximate threshold value, 1.03, was reached for curve $4 \mathrm{~B}$, which is the reflectance spectrum for the device designed in this work. Curve 4D is the target reflectance spectrum used in the optimization.

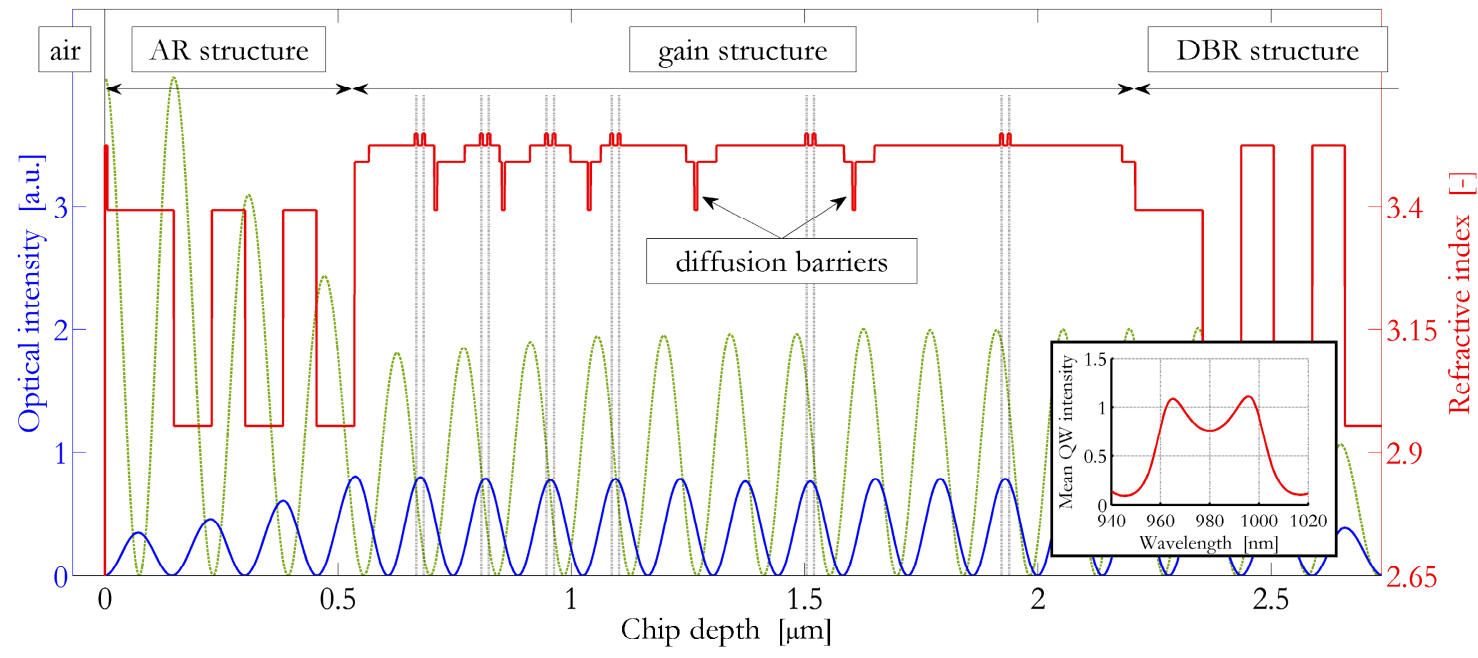

Figure 5. Simulations of the optical field inside the subcavity and the refractive index of the layer structure. Shown with blue solid line and green dashed line are the optical intensities of the fields at the antiresonant wavelength, $980 \mathrm{~nm}$, and at one of the resonant wavelengths, $960 \mathrm{~nm}$, respectively. The red solid curve is the refractive index of the layers in the structure. The positions of the QWs are also indicated by vertical black dotted lines. Shown in the inset is the wavelength dependency of the average optical intensity in the QWs, illustrating how the misalignment between the antinodes of the subcavity field and the QWs away from the center wavelength is compensated by an increase in the entire subcavity field as the structure becomes more resonant. 


\section{SETUP OF OP-SDL}

The gain element was fabricated using MOVPE and was grown in reverse order. The wafers were metalized and cleaved into $\sim 3 \times 3 \mathrm{~mm}^{2}$ chips, which were soldered directly to copper submounts. The substrate was then removed using wet chemical etching ${ }^{8}$ leaving only a thin film of the necessary layers. Although it might have further improved the heat transport, an intra-cavity heat-spreader was not used since that would possibly have introduced unwanted etalon effects while tuning. The processed chips were mounted on a water-cooled copper heatsink held at $-8^{\circ} \mathrm{C}$ in a linear cavity, see figure 6.

As a wavelength-selective element a birefringent filter (BRF), consisting of a $1.0 \mathrm{~mm}$ thick quartz plate, was used. This was inserted into the cavity with the surface normal of the BRF at the Brewster angle to the optical axis of the cavity in order to minimize reflection losses. As the cavity field enters the BRF it is decomposed into an ordinary and an extraordinary field along the respective axes. Due to the slight difference in refractive index for the fields, a phase retardation between them is introduced, which leads to a change in polarization of the total field at the other surface of the BRF, thus no longer fulfilling the condition for lossless transmission by the Brewster effect. However, for some wavelengths the phase retardation will be an integer multiple of $\pi$, which will preserve the polarization of the field. The Brewster condition can then be upheld at both surfaces, which yields very small losses so that lasing can occur. By rotating the BRF around its optical axis the refractive index of the extraordinary field is changed, which alters the condition for preserved polarization and thus the wavelength of the OP-SDL can be tuned.

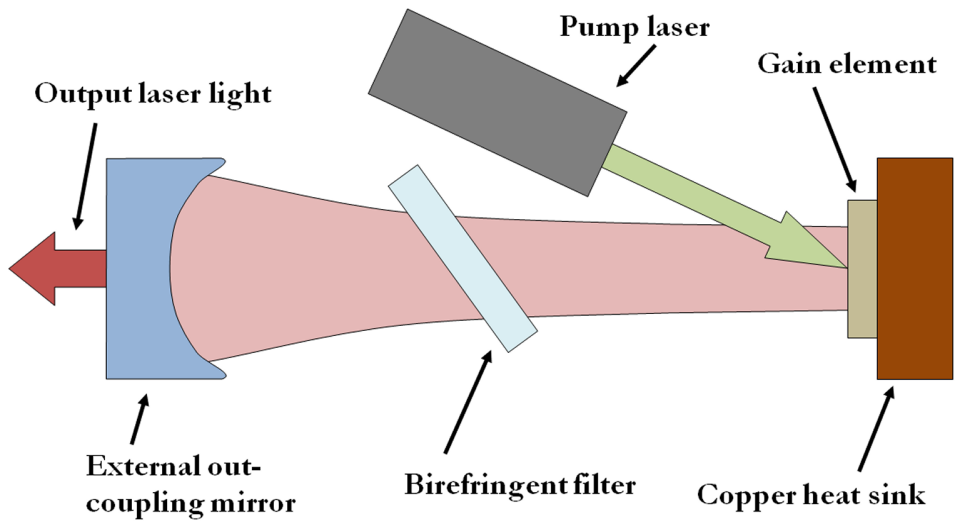

Figure 6. Schematic view of the OP-SDL setup. 


\section{EXPERIMENTS}

Even though the spectral reflectance of the gain element was the quantity used as the quality measure in the design, the simulations were validated by comparing instead the simulated and measured threshold pump intensities. The reason for this is that the direct measurement of the spectral reflectance of the gain element under pump excitation is a more difficult experiment since the laser probe must then come from an external tunable laser, rather than being generated in the OP-SDL. The results of the pump threshold measurements together with the corresponding results from the simulations are shown in figure 7. As can be seen there is a strong agreement between measurements and simulations, showing that the physical models we have used are reasonable and account for all relevant effects. From figure 7 it is also clear that the optimized design is indeed more broadband than is a conventional, narrowband OP-SDL, which is a strong indication that the design strategy for broadband operation is effective.

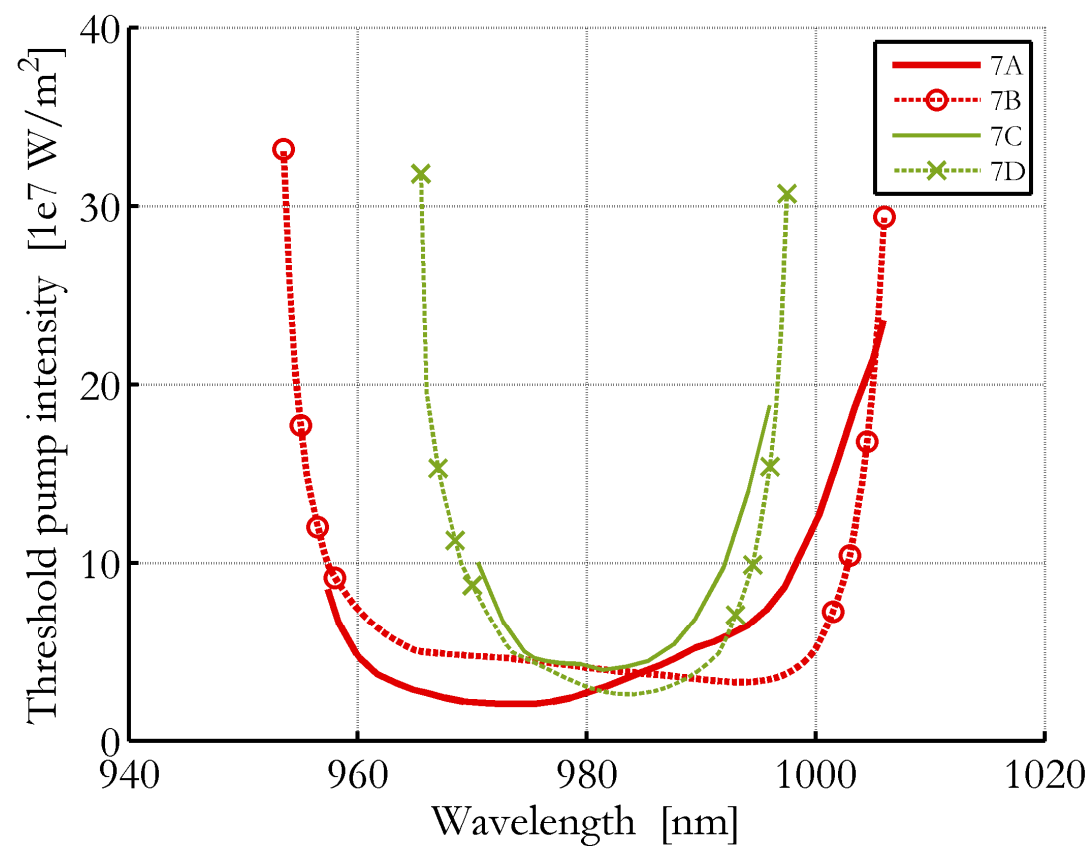

Figure 7. Threshold pump intensity as a function of wavelength for the gain element designed for broadband operation (7A \& 7B) and also for a conventional, narrowband gain element (7C \& 7D). Measurements are shown with solid lines (7A $\& 7 \mathrm{C}$ ) and simulation results with dashed lines (7B \& 7D; markers are included only at the edge wavelengths to increase clarity).

High-power tuning experiments with the designed gain element were also performed and figure 8 shows the power spectrum of the OP-SDL for two different pump powers. At the maximum available pump power, $16.7 \mathrm{~W}$, the OP-SDL could be continuously tuned over $43 \mathrm{~nm}$ (with a $27 \mathrm{~nm} 3 \mathrm{~dB}$ tuning range), corresponding to a rotation of the $\mathrm{BRF}$ by $\sim 8$ degrees, and the maximum output power was $2.6 \mathrm{~W}$ (curve $8 \mathrm{~A}$ ). For comparison the power spectrum at a pump power of $11.0 \mathrm{~W}$ is also shown. The tuning range for this pump power was $42 \mathrm{~nm}(31 \mathrm{~nm} 3 \mathrm{~dB}$ tuning range) and the maximum output power was $1.5 \mathrm{~W}$ (curve $8 \mathrm{~B}$ ). The reflection loss from the BRF was between $0.1 \%$ and $0.2 \%$ according to measurements of the power reflected from the BRF surfaces.

It is interesting to note that the tuning range is not significantly expanded by the increase in pump power and the $3 \mathrm{~dB}$ tuning range is even somewhat reduced. The reason is most likely related to thermal roll-over and other performance-degrading effects resulting from the temperature increase. The redshift of the power spectrum at higher pump powers is related to the temperature shift of the subcavity resonance wavelength and of the peak wavelength for optical gain in the QWs. 


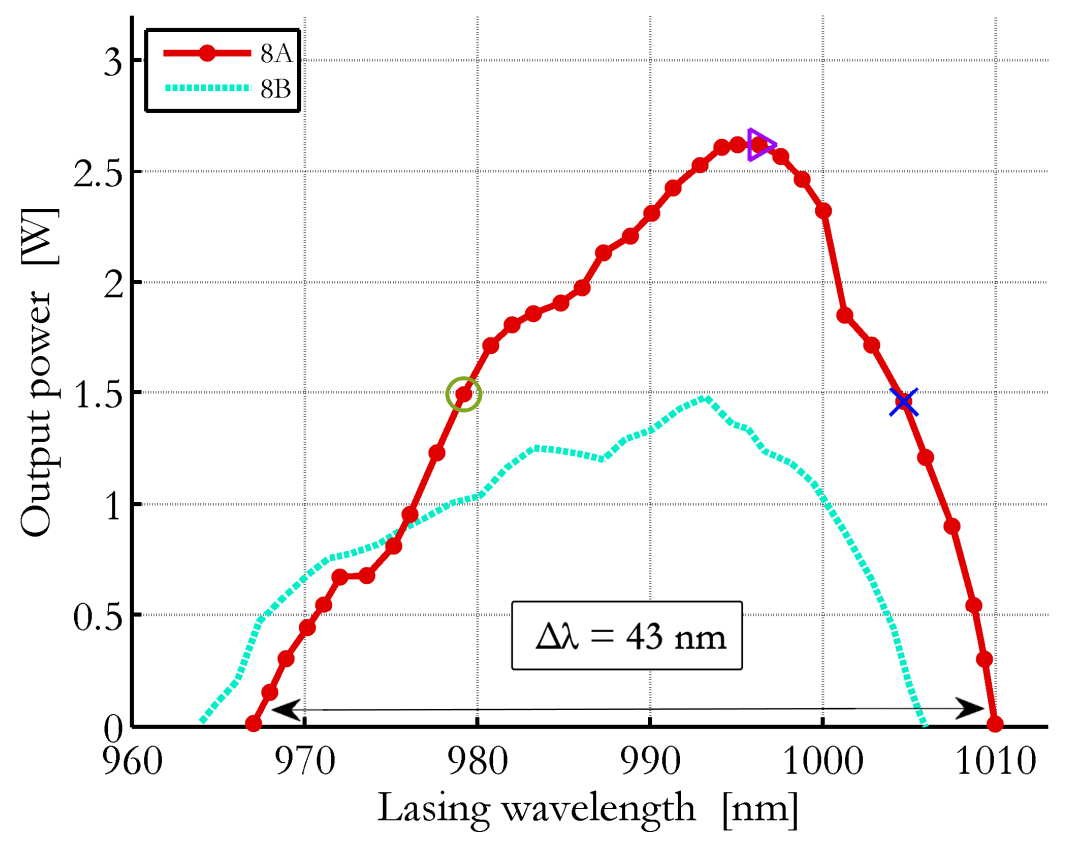

Figure 8. Measured power spectra of the OP-SDL with broadband gain element at two (high) values for the incident pump power. For curve $8 \mathrm{~A}$ the tuning range was $43 \mathrm{~nm}$ at an incident pump power of $16.7 \mathrm{~W}$. For curve $8 \mathrm{~B}$ the tuning range was $42 \mathrm{~nm}$ at an incident pump power of $11.0 \mathrm{~W}$.

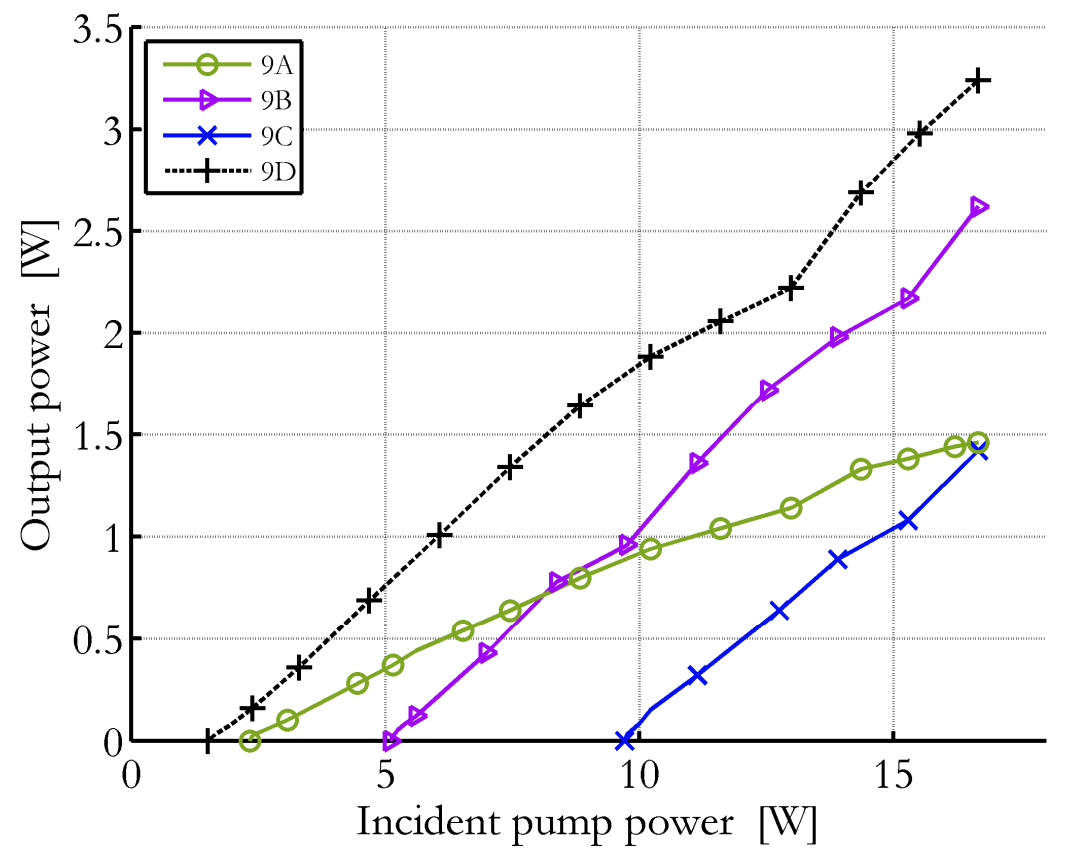

Figure 9. Output vs. incident pump power characteristics of the OP-SDL. Curves 9A, 9B, and 9C show the characteristics when the OP-SDL was tuned to wavelengths of $979 \mathrm{~nm}, 996 \mathrm{~nm}$, and $1005 \mathrm{~nm}$, respectively, and curve 9D shows the characteristics for the free-running OP-SDL. 
The output vs. incident pump power characteristics are shown in figure 9 for the OP-SDL tuned to three different wavelengths as well as for the free-running OP-SDL, i.e. without the BRF inserted. The maximum output power for the free-running laser (without the BRF) was $3.2 \mathrm{~W}$ (limited by the available pump power) and the slope efficiency was 0.21 . The three wavelengths represent the maximum output power (9B) and an output power of $\sim 1.5 \mathrm{~W}$ (9A and 9C), respectively, as can be seen from figure 8. As mentioned, increasing the pump induces an increase in temperature, which moves the power spectrum towards longer wavelengths. This explains the variation in slope efficiency with the lasing wavelength as can be seen in figure 9; with increasing pump power the power spectrum will move away from the short wavelength side but towards the long wavelength side.

\section{CONCLUSION}

We have shown that it is possible to achieve broadband reflectance that is nearly constant at some prescribed value larger than unity from an OP-SDL gain element with identical QWs. The strategy in designing the layer structure of the gain element has been to make the subcavity antiresonant at the center wavelength, with the two nearest resonance wavelengths - whose spacing essentially limits the bandwidth - sufficiently far from the center wavelength even when using the long subcavity needed to contain the large number (12) of QWs. Moreover, an AR structure was used to finetune the spectral behavior. The gain structure was parametrically optimized using the distribution of the QWs and the thicknesses, compositions, and number of layers in the AR structure as variables to produce a reflectance spectrum as close as possible to the target reflectance spectrum.

A designed gain element was fabricated and the spectral threshold pump intensity was measured, showing a strong agreement with simulations. This also validated the design strategy, since the low-threshold pump intensity regime for this gain element was as wide as $\sim 49 \mathrm{~nm}$, as compared to $\sim 25 \mathrm{~nm}$ for a conventional, narrowband gain element. Furthermore, high-power tuning experiments were performed showing a wide tuning range of $43 \mathrm{~nm}(4.3 \%$ relative tuning) with a maximum output power of $2.6 \mathrm{~W}$, which to our knowledge is the best combination of tuning range and output power for an OP-SDL of this type.

\section{REFERENCES}

[1] Rudin, B., Rutz, A., Hoffmann, M., Maas, D. J. H. C., Bellancourt, A., Gini, E., Südmeyer, T. and Keller, U., "Highly efficient optically pumped vertical-emitting semiconductor laser with more than $20 \mathrm{~W}$ average output power in a fundamental transverse mode," Opt. Lett. 33(22), 2719--2721 (2008).

[2] Chilla, J., Shu, Q., Zhou, H., Weiss, E., Reed, M. and Spinelli, L., "Recent advances in optically pumped semiconductor lasers," Proc. SPIE Int. Soc. Opt. Eng. 6451, 645109--10 (2007).

[3] Demaria, F., Lorch, S., Menzel, S., Riedl, M., Rinaldi, F., Rösch, R. and Unger, P., "Design of Highly Efficient HighPower Optically Pumped Semiconductor Disk Lasers," IEEE J. Sel. Topics in Quantum Electron. 15(3), 973--977 (2009).

[4] Garnache, A., Kachanov, A. A., Stoeckel, F. and Houdré, R., "Diode-pumped broadband vertical-external-cavity surface-emitting semiconductor laser applied to high-sensitivity intracavity absorption spectroscopy," J. Opt. Soc. Am. B 17(9), 1589--1598 (2000).

[5] Fan, L., Fallahi, M., Zakharian, A., Hader, J., Moloney, J., Bedford, R., Murray, J., Stolz, W. and Koch, S., "Extended Tunability in a Two-Chip VECSEL," IEEE Photonic. Tech. L. 19(8), 544--546 (2007).

[6] Paajaste, J., Suomalainen, S., Koskinen, R., Härkönen, A., Guina, M. and Pessa, M., "High-power and broadly tunable GaSb-based optically pumped VECSELs emitting near 2 m," Journal of Crystal Growth 311(7), 1917--1919 (2009).

[7] Geske, J., Gan, K., Okuno, Y., Piprek, J. and Bowers, J., "Vertical-cavity surface-emitting laser active regions for enhanced performance with optical pumping," IEEE J. Quantum. Electron. 40(9), 1155--1162 (2004).

[8] Häring, R., Paschotta, R., Aschwanden, A., Gini, E., Morier-Genoud, F. and Keller, U., "High-power passively mode-locked semiconductor lasers," IEEE J. Quantum. Electron. 38(9), 1268--1275 (2002). 